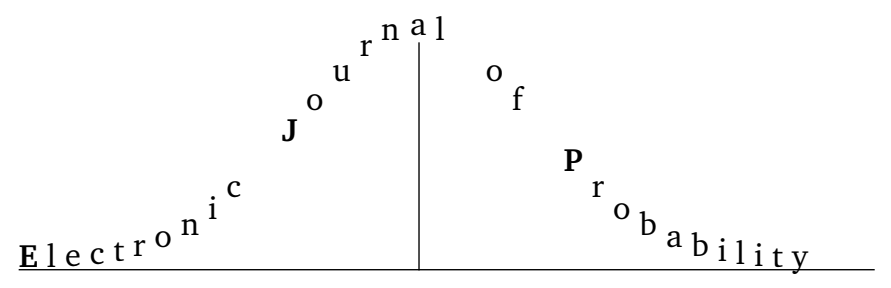

Vol. 14 (2009), Paper no. 57, pages 1705-1726.

Journal URL

http://www.math.washington.edu/ ejpecp/

\title{
Spontaneous breaking of continuous rotational symmetry in two dimensions
}

\author{
Franz Merkl \\ Mathematical Institute \\ University of Munich \\ Theresienstr. 39 \\ D-80333 Munich, Germany \\ Email: merkl@mathematik.uni-muenchen.de \\ Silke W.W. Rolles \\ Zentrum Mathematik, Bereich M5 \\ Technische Universität München \\ D-85747 Garching bei München, Germany \\ Email: srolles@ma.tum.de
}

\begin{abstract}
In this article, we consider a simple model in equilibrium statistical mechanics for a twodimensional crystal without defects. In this model, the local specifications for infinite-volume Gibbs measures are rotationally symmetric. We show that at sufficiently low, but positive temperature, rotational symmetry is spontaneously broken in some infinite-volume Gibbs measures.
\end{abstract}

Key words: Gibbs measure, rotation, spontaneous symmetry breaking, continuous symmetry.

AMS 2000 Subject Classification: Primary 60K35; Secondary: 82B20, 82B21.

Submitted to EJP on June 21, 2007, final version accepted July 1, 2009. 


\section{Introduction}

According to the famous Mermin-Wagner theorem and its more recent variants, Gibbs equilibrium distributions of thermodynamic systems in two dimensions usually preserve continuous symmetries of their local specifications. This holds, under weak conditions, for the preservation of translational symmetry and for inner symmetries like spin rotations. Important results concerning preservation of continuous symmetries in two dimensions have been proven by Mermin and Wagner [MW66], Dobrushin and Shlosman [DS75], Fröhlich and Pfister [FP81], see also[FP86], Pfister [Pfi81], and by Georgii [Geo99]. Over the decades, the regularity conditions on the interaction potential in Mermin-Wagner type theorems have been astonishingly weakened. Ioffe, Shlosman, and Velenik [ISV02] have proven the absence of continuous symmetry breaking in two-dimensional lattice systems without any smoothness assumptions on the interaction. Recently, a general version of this preservation of symmetries has been shown by Richthammer in [Ric05] and [Ric09], using only very weak regularity assumptions for the local Hamiltonians. This includes, among others, systems of hard disks in two dimensions at any chemical potential.

On the other hand, theorems of Mermin-Wagner type [MW66] are not applicable to spatial rotations in two dimensions. For example, it is conjectured that not all Gibbsian equilibrium distributions describing hard disks in two dimensions at high chemical potentials are rotationally symmetric. Already Fröhlich and Pfister [FP81] remarked that

(...) the breaking of rotation-invariance, i.e. directional ordering, is possible, in principle, in two-dimensional systems with connected correlations which do not fall off more rapidly than the inverse square distance (...)

In this paper, it will be shown that some infinite-volume Gibbsian lattice particle systems in two dimensions indeed show spontaneous breaking of spatial rotational symmetry at low temperature. This symmetry breaking is shown for a system of two-dimensional "atoms". In this system, the atoms are characterized by their location in the plane and their orientation. The local Hamiltonians are chosen rotationally symmetric. Nevertheless, the particle configurations are indexed by a triangular lattice. Thus, the permitted particle configurations may be viewed as a deformed triangular lattice.

Motivation. In the physics literature, spontaneous breaking of rotational symmetry in twodimensional solids has been taken as a fact already a long time ago; see e.g. [Mer68] in the case of a two-dimensional harmonic net. Even more, in the physics literature, melting transitions of two-dimensional solids and liquid and "hexatic" phases have been discussed, see e.g. [NH79].

However, from a fundamental, statistical mechanical point of view, the difference between crystalline solids and fluids is not well understood rigorously. The remarkable recent paper [BLRW06] shows the existence of a solidification phase transition for interacting tiles with zippers. With the exception of this reference, we are not aware of any other proof of a melting/freezing phase transition in a continuum system of interacting particles in thermal equilibrium. Even worse, there is no proof of crystalline order at low, but positive temperature for any realistic continuum particle system. It seems reasonable to take the breaking of rotational symmetry as one characteristic property of crystals. A goal in the long run is to understand models for crystallization of increasing complexity from a fundamental point of view. As a starting point, we examine a simplified model for a two-dimensional crystal. We exclude defects of the crystal by definition of the model, taking 
an infinite system of particles that looks locally like a slightly perturbed triangular lattice, up to perturbations of size $\varepsilon>0$. Intuitively, one may think of $\varepsilon$ being small, although this is not required in our model. The only reason why we work with the triangular lattice instead of square lattices is that we think that the triangular lattice may be a more realistic model for ordered two-dimensional particle systems. Our proof of spontaneous breaking of rotational symmetry in this model relies on a simple variant of infrared bounds; no reflection positivity is required.

The goal of this paper is to understand some features of two-dimensional crystals at low temperature in a simplified model. It tells us nothing about melting/freezing phase transitions. We do not know the high-temperature behaviour of the model discussed in this paper. It may be possible that for small $\varepsilon>0$, it shows spontaneous breaking of rotational symmetry at all temperatures, in other words, it might have no melting transition. One may compare it with the following: For a spin model with $\mathrm{O}(2)$-invariant ferromagnetic interaction with a constraint that enforces approximate local alignment of the spins, Aizenman [Aiz94] proves a power-law lower bound for the correlation function at all temperatures. Similar to the Patrascioiu-Seiler model studied by Aizenman [Aiz94], our model forbids topological defects by using appropriate constraints. The present model also forbids lattice defects. It is a major task for future work to remove these constraints.

\section{Results}

Definition of the model. Let $\tau:=e^{\pi i / 3}$, and consider the triangular lattice $\mathscr{T}=(V, E)$ with vertex set $V:=\mathbb{Z}+\tau \mathbb{Z}$ and set of directed edges $E:=\{(u, v): u, v \in V$ with $|u-v|=1\}$. In particular, there are two directed edges with opposite directions between any pair of vertices at distance one from each other.

We consider an infinite system of atoms, indexed by the vertex set $V$ of the triangular lattice. Intuitively speaking, every atom has six "arms". At the end of each arm, there is a "sticky ball" of radius $\varepsilon$, where $\varepsilon>0$ is fixed (see Figure 1).

Figure 1: An atom consisting of 6 arms with sticky balls at the ends of the arms.

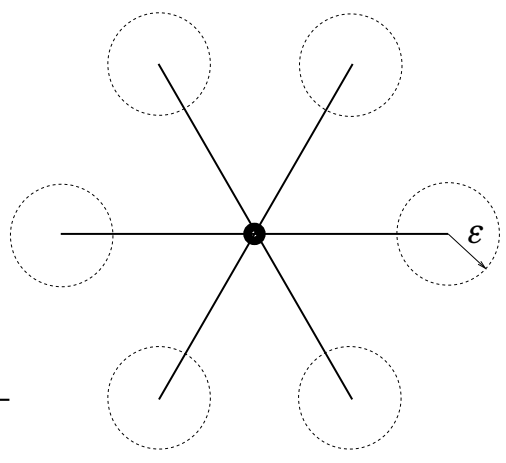

At each of the six sticky balls, another atom is located. The neighboring atom is located somewhere inside the ball of radius $\varepsilon$; it has again six sticky arms. In Figure 2, three atoms sticking together are shown. 
Figure 2: A molecule consisting of three atoms.

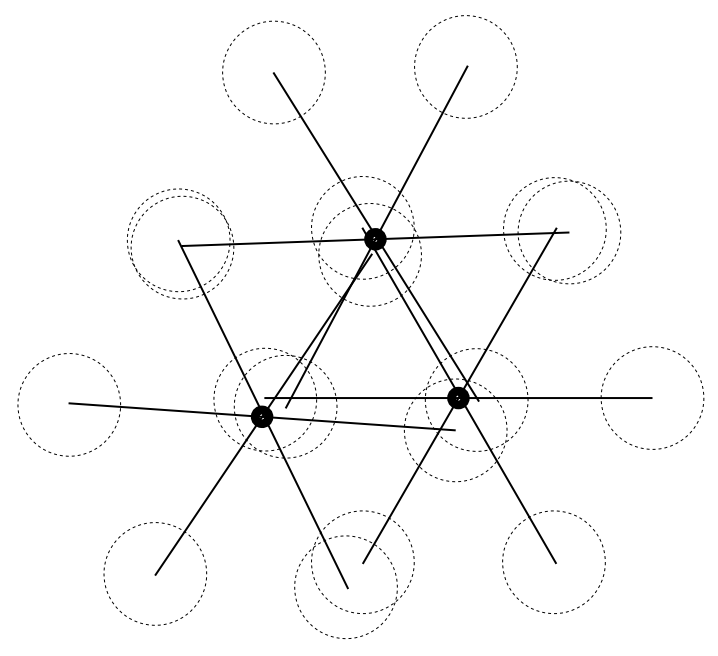

Formally, for $\Lambda \subseteq V$, define the space $\Omega_{\Lambda}$ of configurations on $\Lambda$ by

$$
\Omega_{\Lambda}:=\left\{\left(x_{u}, \gamma_{u}\right)_{u \in \Lambda} \in\left(\mathbb{C} \times S^{1}\right)^{\Lambda}: x_{0}=0\right\}
$$

if $0 \in \Lambda$, and $\Omega_{\Lambda}:=\left(\mathbb{C} \times S^{1}\right)^{\Lambda}$ otherwise. Thus, a configuration consists of the position $x_{u} \in \mathbb{C}$ of the atom with index $u$ and its orientation $\gamma_{u} \in S^{1}=\{z \in \mathbb{C}:|z|=1\}$ for every $u \in \Lambda$. The condition $x_{0}=0$ is a reference fixation of the configurations. We write

$$
\gamma_{u}=e^{i \alpha_{u}} \quad \text { with } \quad-\pi<\alpha_{u} \leq \pi .
$$

A configuration in $\Omega_{\Lambda}$ is of the form

$$
\omega_{\Lambda}=\left(\omega_{u}\right)_{u \in \Lambda} \quad \text { with } \quad \omega_{u}:=\left(x_{u}, \gamma_{u}\right) .
$$

For disjoint $\Lambda, \Lambda^{\prime} \subseteq V$, set $\omega_{\Lambda} \omega_{\Lambda^{\prime}}:=\omega_{\Lambda} \cup \omega_{\Lambda^{\prime}}$. We endow $\Omega_{\Lambda}$ with the $\sigma$-field $\mathscr{F}_{\Lambda}$ generated by the projections $\omega_{\Lambda} \mapsto x_{u}$ and $\omega_{\Lambda} \mapsto \gamma_{u}$ to the individual coordinates and the reference measure

$$
\rho_{\Lambda}\left(d \omega_{\Lambda}\right):= \begin{cases}\delta_{0}\left(d x_{0}\right) \prod_{u \in \Lambda \backslash\{0\}} d x_{u} \prod_{v \in \Lambda} d \gamma_{v} & \text { for } 0 \in \Lambda, \\ \prod_{u \in \Lambda} d x_{u} \prod_{v \in \Lambda} d \gamma_{v} & \text { otherwise }\end{cases}
$$

here $\delta_{0}$ denotes the unit point mass in 0 , and $d x_{u}$ and $d \gamma_{v}$ denote the Lebesgue measure on $\mathbb{C}$ and $S^{1}$, respectively.

Let $\varepsilon>0$ be a fixed perturbation parameter, and let $h: \mathbb{C} \rightarrow[0, \infty]$ be a potential with the following properties:

- $h$ is measurable and Lebesgue-almost everywhere continuous.

- $h$ is rotationally symmetric: $h(a)=h(b)$ for $|a|=|b|$.

- For all $a \in \mathbb{C}$,

$$
h(a) \geq|a|^{2}+\infty \cdot 1_{\{|a| \geq \varepsilon\}}
$$


- Furthermore,

$$
c_{1}\left(\varepsilon^{\prime}\right):=\sup _{a \in \mathbb{C}:|a|<\varepsilon^{\prime}} h(a)
$$

satisfies

$$
c_{1}(\varepsilon)<\infty \quad \text { and } \quad \lim _{\varepsilon^{\prime} \rightarrow 0} c_{1}\left(\varepsilon^{\prime}\right)=0
$$

One possible choice for the potential is the function $h(a)=|a|^{2}+\infty \cdot 1_{\{|a| \geq \varepsilon\}}$.

Let $\Lambda \subset V$ be a finite set containing 0 with outer boundary $\partial \Lambda$ and set $\bar{\Lambda}:=\Lambda \cup \partial \Lambda$. For $\omega_{\Lambda} \in \Omega_{\Lambda}$ and $\omega_{\partial \Lambda} \in \Omega_{\partial \Lambda}$ with $\omega_{\Lambda} \omega_{\partial \Lambda}=\left(x_{u}, \gamma_{u}\right)_{u \in \bar{\Lambda}}$, define the local Hamiltonian with boundary condition $\omega_{\partial \Lambda}$ by

$$
H_{\Lambda}\left(\omega_{\Lambda} \mid \omega_{\partial \Lambda}\right):=\sum_{u, v \in \Lambda:(u, v) \in E} h\left(x_{u}-x_{v}-\gamma_{u}(u-v)\right)
$$

The partition sum associated with this local Hamiltonian at the inverse temperature $\beta>0$ is given by

$$
Z_{\beta, \Lambda}\left(\omega_{\partial \Lambda}\right):=\int_{\Omega_{\Lambda}} e^{-\beta H_{\Lambda}\left(\omega_{\Lambda} \mid \omega_{\partial \Lambda}\right)} \rho_{\Lambda}\left(d \omega_{\Lambda}\right)
$$

with the convention $e^{-\infty}=0$. Note that $Z_{\beta, \Lambda}$ takes finite values.

Clearly, for $\omega_{\Lambda} \omega_{\partial \Lambda}=\left(x_{u}=u, \gamma_{u}=1\right)_{u \in \bar{\Lambda}}$, the local Hamiltonian is finite. This configuration has an atom with "standard orientation" at every $u \in \Lambda$; i.e. the atoms are located at the vertices of the triangular lattice. All other configurations with finite energy may be viewed as perturbations of this triangular lattice configuration.

For $\omega_{\partial \Lambda} \in \Omega_{\partial \Lambda}$ such that $Z_{\beta, \Lambda}\left(\omega_{\partial \Lambda}\right)>0$, the finite-volume Gibbs measure at the temperature $T=$ $1 / \beta$ with the boundary condition $\omega_{\partial \Lambda}$ is defined to be the following probability measure on $\Omega_{\Lambda}$ :

$$
\mu_{\beta, \Lambda}\left(d \omega_{\Lambda} \mid \omega_{\partial \Lambda}\right):=\frac{e^{-\beta H_{\Lambda}\left(\omega_{\Lambda} \mid \omega_{\partial \Lambda}\right)}}{Z_{\beta, \Lambda}\left(\omega_{\partial \Lambda}\right)} \rho_{\Lambda}\left(d \omega_{\Lambda}\right)
$$

A probability measure $\mu_{\beta}$ on $\left(\Omega_{V}, \mathscr{F}_{V}\right)$ is called an infinite-volume Gibbs measure at inverse temperature $\beta$, if it satisfies the DLR-conditions:

$$
\mu_{\beta}\left(A \mid \omega_{\Lambda^{c}}\right)=\frac{1}{Z_{\beta, \Lambda}\left(\omega_{\partial \Lambda}\right)} \int_{\Omega_{\Lambda}} 1_{A}\left(\tilde{\omega}_{\Lambda} \omega_{\Lambda^{c}}\right) e^{-\beta H_{\Lambda}\left(\tilde{\omega}_{\Lambda} \mid \omega_{\partial \Lambda}\right)} \rho_{\Lambda}\left(d \tilde{\omega}_{\Lambda}\right) \quad \mu_{\beta} \text {-a.s. }
$$

for any $A \in \mathscr{F}_{V}$ and any finite $\Lambda \subset V$ containing 0 . In particular, this includes the requirement $Z_{\beta, \Lambda}\left(\omega_{\partial \Lambda}\right)>0$ for $\mu_{\beta}$-almost every $\omega \in \Omega_{V}$. 
Rotational symmetry of the local specifications. For $s \in S^{1}$ and any $\Lambda \subseteq V$, define the rotation $R_{s}: \Omega_{\Lambda} \rightarrow \Omega_{\Lambda},\left(x_{u}, \gamma_{u}\right)_{u \in \Lambda} \mapsto\left(s x_{u}, s \gamma_{u}\right)_{u \in \Lambda}$. For finite $\Lambda \subset V$ with $0 \in \Lambda$, note that both, the reference measures $\rho_{\Lambda}$ and the local Hamiltonians, are invariant with respect to $R_{s}$ :

$$
H_{\Lambda}\left(R_{s} \omega_{\Lambda} \mid R_{s} \omega_{\partial \Lambda}\right)=H_{\Lambda}\left(\omega_{\Lambda} \mid \omega_{\partial \Lambda}\right), \quad\left(\omega_{\Lambda} \in \Omega_{\Lambda}, \omega_{\partial \Lambda} \in \Omega_{\partial \Lambda}\right)
$$

An infinite-volume Gibbs measure $\mu_{\beta}$ is called rotationally invariant, if for all $s \in S^{1}$, the image measure $R_{s}\left[\mu_{\beta}\right]$ of $\mu_{\beta}$ under the map $R_{s}$ equals $\mu_{\beta}$. Otherwise, we say that $\mu_{\beta}$ spontaneously breaks the rotational symmetry.

Theorem 2.1 (Spontaneous breaking of rotational symmetry). Let $\varepsilon>0$. At all sufficiently small temperatures $\beta^{-1}>0$, there exists an infinite-volume Gibbs measure $\mu_{\beta}$ that spontaneously breaks the rotational symmetry. More precisely,

$$
E_{\mu_{\beta}}\left[\alpha_{0}\right]=0 \text { and } \lim _{\beta \rightarrow \infty} \operatorname{Var}_{\mu_{\beta}}\left(\alpha_{0}\right)=0,
$$

so that for all sufficiently low temperatures $\beta^{-1}$, with respect to $\mu_{\beta}$, the orientation $\gamma_{0}$ of the 0 -th particle cannot be uniformly distributed on $S^{1}$.

Nevertheless, there is a homotopy between the standard configuration and a configuration that equals the standard configuration near infinity, but is rotated by an arbitrarily large angle on any fixed finite piece. The following remark states this more formally. Its proof is sketched at the end of Section 4.

Remark 2.2 (Arbitrary rotations of finite pieces). For every $\varepsilon>0$, every finite set $\Lambda \subset V$, and every $\varphi>0$, there is a path $\left(x_{u}(t), \gamma_{u}(t)\right)_{u \in V,-\varphi \leq t \leq \varphi}$ in $\Omega_{V}$ with the following properties:

(a) Standard configuration at deformation parameter $t=0$ : For all $u \in V, x_{u}(0)=u$ and $\gamma_{u}(0)=1$.

(b) Admissible configurations: For all $t \in[-\varphi, \varphi]$, the configuration $\left(x_{u}(t), \gamma_{u}(t)\right)_{u \in V}$ is admissible, i.e. for all $(u, v) \in E$, the bound $\left|x_{u}-x_{v}-\gamma_{u}(u-v)\right|<\varepsilon$ holds.

(c) Continuity: For all $u \in V$, the map $t \mapsto\left(x_{u}(t), \gamma_{u}(t)\right)$ is continuous.

(d) Rotation on $\Lambda$ : For all $u \in \Lambda$ and $t \in[-\varphi, \varphi]$, we have $x_{u}(t)=e^{i t} u$ and $\gamma_{u}(t)=e^{i t}$.

(e) Standard configuration near $\infty$ : There is a finite set $\Sigma$ with $\Lambda \subseteq \Sigma \subset V$, such that for all $u \in V \backslash \Sigma$ and all $t \in[-\varphi, \varphi]$, one has $x_{u}(t)=u$ and $\gamma_{u}(t)=1$.

Finite-volume Gibbs measures with periodic boundary conditions. For $N \in \mathbb{N}$, let $\Lambda_{N}$ be a set of representatives for $(\mathbb{Z}+\tau \mathbb{Z}) /(N \mathbb{Z}+N \tau \mathbb{Z})$ with $0 \in \Lambda_{N}$. More specifically, we take

$$
\Lambda_{N}=\{k+\tau l: k, l \in\{\lfloor-N / 2\rfloor, \ldots,\lfloor N / 2\rfloor-1\}\} .
$$

We introduce the space of all spatially periodic configurations with respect to $N$ :

$$
\Omega_{N}^{\text {per }}:=\left\{\begin{array}{l}
\left(x_{u}, \gamma_{u}\right)_{u \in V} \in\left(\mathbb{C} \times S^{1}\right)^{V}: x_{0}=0, x_{u+l}=x_{u}+l, \gamma_{u+l}=\gamma_{u} \text { for all } \\
u \in V, l \in N V
\end{array}\right\} .
$$


Note that the average density of particles for these configurations is just the same as in the standard triangular lattice $V$, due to the condition $x_{u+l}=x_{u}+l$ rather than $x_{u+l}=x_{u}+$ const $\cdot l$. Sometimes, it is convenient to identify $\Omega_{N}^{\text {per }}$ with the set $\Omega_{\Lambda_{N}}=\left\{\omega_{\Lambda_{N}}: \omega \in \Omega_{N}^{\text {per }}\right\}$. We will implicitly use this identification below.

For periodic boundary conditions with period $N$, we define the finite-volume Hamiltonian $H_{N}$ in analogy to (2.8)

$$
H_{N}(\omega):=\sum_{u \in \Lambda_{N}} \sum_{\substack{v \in V: \\(u, v) \in E}} h\left(x_{u}-x_{v}-\gamma_{u}(u-v)\right), \quad \omega=\left(x_{u}, \gamma_{u}\right)_{u \in V} \in \Omega_{N}^{\mathrm{per}} .
$$

Note that this definition does not depend on the choice of the set $\Lambda_{N}$ of representatives for $V / N V$. In the special case $h(a)=|a|^{2}+\infty \cdot 1_{\{|a| \geq \varepsilon\}}$, we denote this function $H_{N}$ by $H_{N}^{\text {sqr }}$.

Furthermore, we define the finite-volume Gibbs measure at inverse temperature $\beta$ with periodic boundary conditions to be the following probability measure on $\Omega_{N}^{\mathrm{per}}$ :

$$
\mu_{\beta, N}(d \omega):=\frac{e^{-\beta H_{N}(\omega)}}{Z_{\beta, N}} \rho_{N}(d \omega)
$$

with the partition sum

$$
Z_{\beta, N}:=\int_{\Omega_{N}^{\text {per }}} e^{-\beta H_{N}} d \rho_{N}>0
$$

Here, $\rho_{N}$ denotes the reference measure on $\Omega_{N}^{\text {per }}$ identified with $\rho_{\Lambda_{N}}$ via the identification $\Omega_{N}^{\text {per }} \equiv$ $\Omega_{\Lambda_{N}}$.

The following theorem is a finite-volume analogue of Theorem 2.1. The given bound is uniform in the size of the finite box.

Theorem 2.3 (Finite-volume estimate). For all $\varepsilon>0$ and all $\delta>0$, there exist $\beta_{0}>0$ and $N_{0} \in \mathbb{N}$ such that for all $\beta \in\left(\beta_{0}, \infty\right)$ and all $N \geq N_{0}$, the variance of $\alpha_{0}$ with respect to the Gibbs measure $\mu_{\beta, N}$ is bounded above by $\delta$ :

$$
\operatorname{Var}_{\mu_{\beta, N}}\left(\alpha_{0}\right)<\delta
$$

Theorems 2.1 and 2.3 hold also for slightly different local Hamiltonians. For instance, we could index the atoms by the square lattice instead of the triangular lattice, and our results still hold.

The following theorem provides the link between the infinite-volume result in Theorem 2.1 and the finite-volume estimate in Theorem 2.3 .

Theorem 2.4 (Infinite-volume limit). For all $\varepsilon>0$ and all $\beta>0$, there exists a strictly increasing sequence $\left(N_{k}\right)_{k \in \mathbb{N}}$ of natural numbers such that the finite-dimensional marginals of $\left(\mu_{\beta, N_{k}}\right)_{k \in \mathbb{N}}$ converge weakly to the finite-dimensional marginals of an infinite-volume Gibbs measure $\mu_{\beta}$ as specified by the DLR-conditions (2.11).

In particular, this means that for any $(u, v) \in E$, the inequality $\left|x_{u}-x_{v}-\gamma_{u}(u-v)\right|<\varepsilon$ holds $\mu_{\beta^{-}}$ almost surely. Furthermore, all finite-dimensional marginals of $\mu_{\beta}$ are absolutely continuous with respect to the corresponding reference measures. 
Intuitive ideas behind the proofs. Before we prove our results, let us explain some intuitive ideas in a simpler model. This paragraph serves only to provide some rough intuitive background information without giving detailed proofs. The formal proofs of our theorems in later sections can be checked even if one ignores the intuitive picture.

Let $x_{u}^{0}:=u, \alpha_{u}^{0}:=0$, and $\gamma_{u}^{0}:=e^{i \alpha_{u}^{0}}=1$ denote the coordinates of the "standard configuration" with one particle with standard orientation at every vertex $u$. We set $y_{u}:=x_{u}-x_{u}^{0}=x_{u}-u$. Note that $\alpha_{u}=\alpha_{u}-\alpha_{u}^{0}$. Let $\langle w, z\rangle=\operatorname{Re}(w \bar{z})$ denote the Euclidean scalar product of $w, z \in \mathbb{C}$.

Let us consider the second order Taylor approximation at $x^{0}$ and $\alpha^{0}$ of the Hamiltonian $H_{N}^{\text {sqr }}$ viewed as a function of $x$ and $\alpha$. In these variables, the Hamiltonian of the linearized model is given by

$$
H_{N}^{\text {gauss }}(y, \alpha):=\sum_{u \in \Lambda_{N}} \sum_{\substack{v \in V: \\(u, v) \in E}}\left\{\left|y_{u}-y_{v}\right|^{2}-2\left\langle y_{u}-y_{v}, i(u-v)\right\rangle \alpha_{u}+\alpha_{u}^{2}\right\}
$$

for $N$-periodic configurations $(y, \alpha)=\left(y_{u}, \alpha_{u}\right)_{u \in V} \in(\mathbb{C} \times \mathbb{R})^{V}$. The corresponding local Hamiltonians for the infinite-volume system are invariant with respect to the linearized rotations

$$
\tilde{R}_{t}:\left(y_{u}, \alpha_{u}\right)_{u} \mapsto\left(y_{u}+t i u, \alpha_{u}+t\right)_{u}
$$

for all $t \in \mathbb{R}$. However, since these linearized rotations do not preserve periodic boundary conditions, they are no symmetries of the model in finite volume with periodic boundary conditions.

Let

$$
V^{*}:=\{p \in \mathbb{C}:\langle p, v\rangle \in 2 \pi \mathbb{Z} \text { for all } v \in V\}=\tau_{1}^{*} \mathbb{Z}+\tau_{2}^{*} \mathbb{Z},
$$

where $\tau_{1}^{*}=2 \pi(1-i / \sqrt{3})$ and $\tau_{2}^{*}=4 \pi i / \sqrt{3}$, denote the dual lattice to $V$. Furthermore, let $\Lambda_{N}^{*}$ be a set of representatives for $\left(N^{-1} V^{*}\right) \bmod V^{*}$ with $0 \in \Lambda_{N}^{*}$. For example, one may take

$$
\Lambda_{N}^{*}=\left\{N^{-1} m_{1} \tau_{1}^{*}+N^{-1} m_{2} \tau_{2}^{*}: m_{1}, m_{2} \in\{0, \ldots, N-1\}\right\} .
$$

We introduce Fourier variables:

$$
\hat{y}_{p}=\left|\Lambda_{N}\right|^{-1} \sum_{u \in \Lambda_{N}} y_{u} e^{-i\langle p, u\rangle} \quad \text { and } \quad \hat{\alpha}_{p}=\left|\Lambda_{N}\right|^{-1} \sum_{u \in \Lambda_{N}} \alpha_{u} e^{-i\langle p, u\rangle}
$$

with the inverse

$$
y_{u}=\sum_{p \in \Lambda_{N}^{*}} \hat{y}_{p} e^{i\langle p, u\rangle} \quad \text { and } \quad \alpha_{u}=\sum_{p \in \Lambda_{N}^{*}} \hat{\alpha}_{p} e^{i\langle p, u\rangle} .
$$

Because of translational symmetry, the Fourier-transform block-diagonalizes the Hamiltonian $H_{N}^{\text {gauss }}$ : Define the matrix $A_{p}$ for all $p \in \mathbb{C}$ by

$$
A_{p}=\sum_{l \in \mathscr{N}}\left(\begin{array}{ll}
\left|1-e^{i\langle p, l\rangle}\right|^{2} & l\left(1-e^{-i\langle p, l\rangle}\right) \\
\bar{l}\left(1-e^{i\langle p, l\rangle}\right) & 1
\end{array}\right)
$$

where $\mathscr{N}:=\{l \in V:|l|=1\}$ denotes the set containing the six neighbors of the origin in the triangular lattice. Then, one has for all $N$-periodic $(y, \alpha)$ :

$$
H_{N}^{\text {gauss }}(y, \alpha)=\left|\Lambda_{N}\right| \sum_{p \in \Lambda_{N}^{*}}\left(\overline{\hat{y}}_{p}, \bar{i}_{\hat{\alpha}_{p}}\right) A_{p}\left(\begin{array}{c}
\hat{y}_{p} \\
i \hat{\alpha}_{p}
\end{array}\right) .
$$


The only points where $A_{p}$ is non-invertible, are $p \equiv 0 \bmod V^{*}$; see (3.20), below. Close to the singularity, i.e. in the infrared regime $p \rightarrow 0$, one has

$$
A_{p}=3\left(\begin{array}{cc}
|p|^{2} & i p \\
-i \bar{p} & 2
\end{array}\right)+\text { lower order terms }
$$

and consequently,

$$
A_{p}^{-1}=\frac{1}{3}\left(\begin{array}{ll}
2|p|^{-2} & -i p|p|^{-2} \\
i \bar{p}|p|^{-2} & 1
\end{array}\right)+\text { lower order terms. }
$$

Consequently, if we denote the entries of a $2 \times 2$-matrix $B$ by $B_{j, k}, j, k=1,2$, we obtain

$$
\left(\overline{\hat{y}}_{p}, \bar{i}_{\hat{\alpha}_{p}}\right) A_{p}\left(\begin{array}{l}
\hat{y}_{p} \\
i \hat{\alpha}_{p}
\end{array}\right) \geq \frac{\operatorname{det} A_{p}}{\left(A_{p}\right)_{1,1}}\left|\hat{\alpha}_{p}\right|^{2}=\frac{\left|\hat{\alpha}_{p}\right|^{2}}{\left(A_{p}^{-1}\right)_{2,2}} \geq c_{2}\left|\hat{\alpha}_{p}\right|^{2}
$$

for all $p$ close to 0 with a constant $c_{2}>0$. If $p$ stays bounded away from the singularity $p \equiv$ $0 \bmod V^{*}$, the same bound holds. Hence, unlike the well-known infrared bounds [FSS76] for the classical isotropic Heisenberg model in three or more dimensions, where the two-point function in Fourier space is bounded by $O\left(|p|^{-2}\right)$ as $p \rightarrow 0$, the relevant entry of the covariance matrix in the linearized model is bounded by a constant, uniformly in $p$. This behavior remains the same for the model with Hamiltonian $H_{N}$. The uniform lower bound for the finite-volume Hamiltonians $H_{N}$ is a crucial ingredient of our proofs. We derive it in Section 3. From this bound, we deduce the estimate for the variance of the angle $\alpha_{0}$ with respect to $\mu_{\beta, N}$ stated in Theorem 2.3. In Section 4, we show how to pass to the infinite-volume limit.

Remark on the one-dimensional analogue of this model. We emphasize that the mechanism for spontaneous breaking of rotational symmetry as described above in the linearized model works only in dimension two (or higher dimensions). In one dimension, the linearized model does not exhibit spontaneous breaking of rotational symmetry. Consider a one-dimensional chain of twoarmed molecules in a plane. One obtains the corresponding linearized model from $(2.20)$ by replacing the two-dimensional vertex set $V$ by the one-dimensional line $\mathbb{Z}$ with nearest-neighbour edges and replacing $\Lambda_{N}$ by $\{0, \ldots, N-1\}$. This model has a completely different behavior than its two-dimensional version. Let us sketch why. The representation $(2.27 / 2.26)$ of the linearized Hamiltonian remains valid with $\Lambda_{N}^{*}=\{2 \pi j / N: j=0,1, \ldots, N-1\}$ and $\mathscr{N}=\{-1,+1\}$. However, in the one-dimensional model,

$$
A_{p}=2\left(\begin{array}{ll}
(2 \sin (p / 2))^{2} & i \sin p \\
-i \sin p & 1
\end{array}\right)
$$

which implies

$$
\left(A_{p}^{-1}\right)_{2,2}=\frac{1}{2 \sin ^{2}(p / 2)}=\frac{2}{p^{2}}+O(1) \quad \text { as } p \rightarrow 0 .
$$

In contrast to the two-dimensional model, this is not integrable near 0 . As a consequence, the symmetry of linearized rotations is preserved in the corresponding infinite-volume model. This means 
that the one-dimensional linearized model does not have infinite-volume Gibbs measures unless one pins down one directional coordinate, setting e.g. $\alpha_{0}=0$.

Of course, this analysis tells us nothing about the non-linearized one-dimensional model in a rigorous sense. However, it indicates that if rotational symmetry is broken in the one-dimensional non-linearized model, this is caused by a completely different mechanism, not accessible in the linearization.

\section{Finite-volume arguments}

The aim of this section is to prove Theorem 2.3. Throughout the remainder of the article, we fix $\varepsilon>0$. First, we state two simple properties of the finite-volume Gibbs measure $\mu_{\beta, N}$ with periodic boundary conditions:

Lemma 3.1 (Symmetry properties of the law of $\alpha_{v}$ ). For all $N \in \mathbb{N}, \beta>0$, and $v \in V$, one has

$$
\operatorname{Law}_{\mu_{\beta, N}}\left(\alpha_{v}\right)=\operatorname{Law}_{\mu_{\beta, N}}\left(\alpha_{0}\right) \quad \text { and } \quad E_{\mu_{\beta, N}}\left[\alpha_{v}\right]=0 .
$$

Proof. Let $N \in \mathbb{N}, \beta>0$, and $v \in \Lambda_{N}$. The Hamiltonian $H_{N}$ and the reference measure $\rho_{N}$ are invariant under the translation $\left(x_{u}, \gamma_{u}\right)_{u \in V} \mapsto\left(x_{u+v}-x_{v}, \gamma_{u+v}\right)_{u \in V}$. Since the configurations in $\Omega_{N}^{\text {per }}$ are spatially periodic, the distribution $\mu_{\beta, N}$ is also invariant under this translation. It follows that $\gamma_{v}$ has the same distribution as $\gamma_{0}$ under $\mu_{\beta, N}$. Hence, $\operatorname{Law}_{\mu_{\beta, N}}\left(\alpha_{v}\right)=\operatorname{Law}_{\mu_{\beta, N}}\left(\alpha_{0}\right)$.

Next, since $(u, v) \in E$ iff $(\bar{u}, \bar{v}) \in E$ and $h(a)=h(\bar{a})$ for all $a \in \mathbb{C}$, one obtains

$$
\begin{aligned}
\sum_{u \in \Lambda_{N}} \sum_{\substack{v \in V: \\
(u, v) \in E}} h\left(x_{u}-x_{v}-\gamma_{u}(u-v)\right) & =\sum_{u \in \Lambda_{N}} \sum_{\substack{v \in V: \\
(u, v) \in E}} h\left(x_{\bar{u}}-x_{\bar{v}}-\gamma_{\bar{u}}(\bar{u}-\bar{v})\right) \\
& =\sum_{u \in \Lambda_{N}} \sum_{\substack{v \in V: \\
(u, v) \in E}} h\left(\overline{x_{\bar{u}}}-\overline{x_{\bar{v}}}-\overline{\gamma_{\bar{u}}}(u-v)\right) .
\end{aligned}
$$

Thus, the distribution $\mu_{\beta, N}$ is invariant under the reflection $\left(x_{u}, \gamma_{u}\right)_{u \in V} \mapsto\left(\overline{x_{\bar{u}}}, \overline{\gamma_{\bar{u}}}\right)_{u \in V}$. In particular, $\gamma_{0}$ and $\overline{\gamma_{0}}$ have the same law; hence $\alpha_{0}$ and $-\alpha_{0}$ also have the same law, since there is no mass in $\alpha_{0}=\pi$. Consequently, $E_{\mu_{\beta, N}}\left[\alpha_{0}\right]=0$.

Note that the properties (2.5) and (2.7) of the potential imply that

$$
\left\{H_{N}<\infty\right\}=\left\{\omega \in \Omega_{N}^{\mathrm{per}}:\left|x_{u}-x_{v}-\gamma_{u}(u-v)\right|<\varepsilon \text { for all } u, v \in V \text { with }(u, v) \in E\right\} .
$$

The following lemma states a crucial lower bound for the local Hamiltonian with periodic boundary conditions.

Lemma 3.2 (Bounding the Hamiltonian). Let $c_{3}=8 / \pi^{2}$. For all $N \in \mathbb{N}$ and all $\omega=\left(x_{u}, \gamma_{u}\right)_{u \in V} \in$ $\Omega_{N}^{\mathrm{per}}$, the Hamiltonian satisfies the bound

$$
H_{N}(\omega) \geq c_{3} \sum_{u \in \Lambda_{N}} \alpha_{u}^{2}
$$


After a Fourier transform, this inequality may be viewed as a variant of formula (2.30) for Fourier variables $p$ not necessarily close to 0 .

Proof of Lemma 3.2. It suffices to prove the bound (3.4) on the set $\left\{H_{N}<\infty\right\}$. Recall the definition (2.23) of $\Lambda_{N}^{*}$. We introduce again Fourier variables: For $u \in \Lambda_{N}$, set

$$
\begin{aligned}
& x_{u}-u=: y_{u}=\sum_{p \in \Lambda_{N}^{*}} \hat{y}_{p} e^{i\langle p, u\rangle}, \\
& \gamma_{u}-1=: z_{u}=\sum_{p \in \Lambda_{N}^{*}} \hat{z}_{p} e^{i\langle p, u\rangle} .
\end{aligned}
$$

Inserting these identities, we get for any $u, v \in \Lambda_{N}$

$$
\begin{aligned}
\left|x_{u}-x_{v}-\gamma_{u}(u-v)\right|^{2} & =\left|\left(x_{u}-u\right)-\left(x_{v}-v\right)+\left(\gamma_{u}-1\right)(v-u)\right|^{2} \\
& =\left|y_{u}-y_{v}+z_{u}(v-u)\right|^{2} \\
& =\left|\sum_{p \in \Lambda_{N}^{*}} e^{i\langle p, u\rangle}\left\{\hat{y}_{p}\left(1-e^{i\langle p, v-u\rangle}\right)+\hat{z}_{p}(v-u)\right\}\right|^{2} .
\end{aligned}
$$

Recall that $\mathscr{N}$ denotes the set of neighbors of the origin in the triangular lattice. For $l \in \mathscr{N}, p \in \Lambda_{N}^{*}$, and $u \in \Lambda_{N}$, set

$$
\begin{aligned}
\hat{w}_{p, l} & :=\hat{y}_{p}\left(1-e^{i\langle p, l\rangle}\right)+\hat{z}_{p} l, \\
w_{u, l} & :=\sum_{p \in \Lambda_{N}^{*}} \hat{w}_{p, l} e^{i\langle p, u\rangle} .
\end{aligned}
$$

Inserting these abbreviations in (3.7) yields the following expression for the Hamiltonian $H_{N}$ on $\left\{H_{N}<\infty\right\}$ :

$$
\begin{aligned}
H_{N}(\omega) \geq H_{N}^{\mathrm{sqr}}(\omega) & =\sum_{u \in \Lambda_{N}} \sum_{\substack{v \in V: \\
(u, v) \in E}}\left|x_{u}-x_{v}-\gamma_{u}(u-v)\right|^{2} \\
& =\sum_{u \in \Lambda_{N}} \sum_{\substack{v \in V: \\
(u, v) \in E}}\left|\sum_{p \in \Lambda_{N}^{*}} \hat{w}_{p, v-u} e^{i\langle p, u\rangle}\right|^{2} .
\end{aligned}
$$

If $(u, v) \in E$, then $v-u \in \mathscr{N}$. Using the definition of $w_{u, l}$ and applying Parseval's identity, we obtain

$$
\begin{aligned}
H_{N}^{\mathrm{sqr}}(\omega) & =\sum_{u \in \Lambda_{N}} \sum_{l \in \mathscr{N}}\left|\sum_{p \in \Lambda_{N}^{*}} \hat{w}_{p, l} e^{i\langle p, u\rangle}\right|^{2} \\
& =\sum_{l \in \mathcal{N}} \sum_{u \in \Lambda_{N}}\left|w_{u, l}\right|^{2}=\left|\Lambda_{N}\right| \sum_{l \in \mathcal{N}} \sum_{p \in \Lambda_{N}^{*}}\left|\hat{w}_{p, l}\right|^{2} .
\end{aligned}
$$

Next, we insert in the last expression the definition (3.8) of $\hat{w}_{p, l}$ :

$$
\begin{aligned}
H_{N}^{\mathrm{sqr}}(\omega) & =\left|\Lambda_{N}\right| \sum_{p \in \Lambda_{N}^{*}} \sum_{l \in \mathcal{N}}\left|\hat{y}_{p}\left(1-e^{i\langle p, l\rangle}\right)+\hat{z}_{p} l\right|^{2} \\
& =\left|\Lambda_{N}\right| \sum_{p \in \Lambda_{N}^{*}}\left(\overline{\hat{y}}_{p}, \overline{\hat{z}}_{p}\right) A_{p}\left(\begin{array}{c}
\hat{y}_{p} \\
\hat{z}_{p}
\end{array}\right)
\end{aligned}
$$


with the same positive semidefinite matrix

$$
A_{p}=\sum_{l \in \mathscr{N}}\left(\begin{array}{ll}
\left|1-e^{i\langle p, l\rangle}\right|^{2} & l\left(1-e^{-i\langle p, l\rangle}\right) \\
\bar{l}\left(1-e^{i\langle p, l\rangle}\right) & |l|^{2}
\end{array}\right)
$$

as in (2.26). In order to bound the quadratic form given by $A_{p}$ from below, we rewrite $\left(A_{p}\right)_{1,1}$ and then derive a lower bound for $\operatorname{det} A_{p}$.

Set

$$
\mathscr{N}_{+}:=\left\{1, \tau, \tau^{2}\right\}=\left\{1, \frac{1}{2}(1+i \sqrt{3}), \frac{1}{2}(-1+i \sqrt{3})\right\}
$$

Then, $\mathscr{N}=\mathscr{N}_{+} \cup\left(-\mathscr{N}_{+}\right)$. Note that $\left|1-e^{i\langle p, l\rangle}\right|^{2}=\left|1-e^{i\langle p,-l\rangle}\right|^{2}=2(1-\cos \langle p, l\rangle)$ holds for $l \in \mathscr{N}$. Consequently,

$$
\left(A_{p}\right)_{1,1}=\sum_{l \in \mathscr{N}}\left|1-e^{i\langle p, l\rangle}\right|^{2}=4 \sum_{l \in \mathscr{N}_{+}}(1-\cos \langle p, l\rangle)=4\left(3-\cos s_{1}-\cos s_{2}-\cos s_{3}\right)
$$

with $s_{1}=\langle p, 1\rangle, s_{2}=\langle p, \tau\rangle$, and $s_{3}=\left\langle p, \tau^{2}\right\rangle$.

For the determinant, we get:

$$
\operatorname{det} A_{p}=6\left(A_{p}\right)_{1,1}-\left(A_{p}\right)_{1,2}\left(A_{p}\right)_{2,1} \text {. }
$$

We rewrite the last term in the last equation:

$$
\begin{aligned}
\left(A_{p}\right)_{1,2}\left(A_{p}\right)_{2,1} & =\left|\sum_{l \in \mathscr{N}} l\left(1-e^{-i\langle p, l\rangle}\right)\right|^{2} \\
& =\left|\sum_{l \in \mathscr{N}_{+}} l\left(1-e^{-i\langle p, l\rangle}\right)-l\left(1-e^{-i\langle p,-l\rangle}\right)\right|^{2} \\
& =\left|\sum_{l \in \mathscr{N}_{+}} 2 i l \sin \langle p, l\rangle\right|^{2} \\
& =\left|2 \sin s_{1}+(1+i \sqrt{3}) \sin s_{2}+(-1+i \sqrt{3}) \sin s_{3}\right|^{2} \\
& =\left(2 \sin s_{1}+\sin s_{2}-\sin s_{3}\right)^{2}+3\left(\sin s_{2}+\sin s_{3}\right)^{2} .
\end{aligned}
$$

Expanding the last term and using the estimate $4 x y \leq 2\left(x^{2}+y^{2}\right)$ yields:

$$
\begin{aligned}
& \left(2 \sin s_{1}+\sin s_{2}-\sin s_{3}\right)^{2}+3\left(\sin s_{2}+\sin s_{3}\right)^{2} \\
= & 4\left(\sin ^{2} s_{1}+\sin ^{2} s_{2}+\sin ^{2} s_{3}\right)+4 \sin s_{2} \sin s_{3}+4 \sin s_{1} \sin s_{2}-4 \sin s_{1} \sin s_{3} \\
\leq & 8\left(\sin ^{2} s_{1}+\sin ^{2} s_{2}+\sin ^{2} s_{3}\right) .
\end{aligned}
$$

Observe that $\sin ^{2} x=1-\cos ^{2} x=(1+\cos x)(1-\cos x) \leq 2(1-\cos x)$ holds for all $x \in \mathbb{R}$. Combining (3.17) and (3.18) with this bound yields

$$
\left(A_{p}\right)_{1,2}\left(A_{p}\right)_{2,1} \leq 16\left(3-\cos s_{1}-\cos s_{2}-\cos s_{3}\right)
$$


Inserting (3.15) and (3.19) into (3.16) we get:

$$
\operatorname{det} A_{p} \geq 8\left(3-\cos s_{1}-\cos s_{2}-\cos s_{3}\right)=2\left(A_{p}\right)_{1,1} .
$$

In particular, $\operatorname{det} A_{p}$ and $\left(A_{p}\right)_{1,1}$ can vanish only if $s_{1}, s_{2}$, and $s_{3}$ are integer multiples of $2 \pi$, that is, for $p \in V^{*}$. As a consequence, for all $p \in \Lambda_{N}^{*} \backslash\{0\}$, we have

$$
\frac{1}{\left(A_{p}^{-1}\right)_{2,2}}=\frac{\operatorname{det} A_{p}}{\left(A_{p}\right)_{1,1}} \geq 2 .
$$

Thus, we get the following lower bound for the quadratic form described by $A_{p}$ :

$$
\left(\overline{\hat{y}}_{p}, \overline{\hat{z}}_{p}\right) A_{p}\left(\begin{array}{c}
\hat{y}_{p} \\
\hat{z}_{p}
\end{array}\right) \geq 2\left|\hat{z}_{p}\right|^{2}
$$

for all $p \in \Lambda_{N}^{*}$. For $p \in \Lambda_{N}^{*} \backslash\{0\}$, this is an immediate consequence of (3.21). In the singular case $p=0$, the claim (3.22) is clear:

$$
\left(\overline{\hat{y}}_{0}, \overline{\hat{z}}_{0}\right) A_{0}\left(\begin{array}{c}
\hat{y}_{0} \\
\hat{z}_{0}
\end{array}\right)=6\left|\hat{z}_{0}\right|^{2} \geq 2\left|\hat{z}_{0}\right|^{2} .
$$

We conclude from (3.12) and Parseval's identity that

$$
H_{N}(\omega) \geq 2\left|\Lambda_{N}\right| \sum_{p \in \Lambda_{N}^{*}}\left|\hat{z}_{p}\right|^{2}=2 \sum_{u \in \Lambda_{N}}\left|1-\gamma_{u}\right|^{2} .
$$

Since $\left|1-e^{i \alpha}\right|^{2}=2(1-\cos \alpha) \geq 4 \alpha^{2} / \pi^{2}$ for all $\alpha \in(-\pi, \pi]$, the claim (3.4) follows with $c_{3}=$ $8 / \pi^{2}$.

Lemma 3.3 (Bounding the partition sum). Let $c_{3}$ be as in Lemma 3.2 and $c_{1}$ as in (2.6). For all $\delta>0, \beta>0, N \in \mathbb{N}$, and all $\varepsilon^{\prime} \in\left(0, \pi^{-1 / 2}\right)$ with $c_{1}\left(3 \varepsilon^{\prime}\right)<c_{3} \delta / 48$, the partition sum defined in (2.18) satisfies the following lower bound:

$$
Z_{\beta, N} \geq \exp \left(\left\{c_{4}-\beta c_{3} \delta / 8\right\}\left|\Lambda_{N}\right|\right)
$$

with $c_{4}=c_{4}\left(\varepsilon^{\prime}\right)=\log \left(2 \pi\left(\varepsilon^{\prime}\right)^{3}\right)$.

Note that by (2.7), $c_{1}\left(\varepsilon^{\prime}\right) \rightarrow 0$ as $\varepsilon^{\prime} \rightarrow 0$, and consequently, there exists $\varepsilon^{\prime}$ with the required properties.

Proof of Lemma 3.3. Here is the idea: The contribution of the configurations globally close to the standard configuration suffice to show the claimed lower bound.

Let $\delta>0, \beta>0$, and $N \in \mathbb{N}$. We have

$$
\begin{aligned}
Z_{\beta, N} & \geq \int_{H_{N} \in\left[0, c_{3} \delta\left|\Lambda_{N}\right| / 8\right]} e^{-\beta H_{N}} d \rho_{N} \\
& \geq \exp \left(-\beta c_{3} \delta\left|\Lambda_{N}\right| / 8\right) \rho_{N}\left[H_{N} \in\left[0, c_{3} \delta\left|\Lambda_{N}\right| / 8\right]\right] .
\end{aligned}
$$


Let $\varepsilon^{\prime} \in\left(0, \pi^{-1 / 2}\right)$ with $c_{1}\left(3 \varepsilon^{\prime}\right)<c_{3} \delta / 48$, and define

$$
U_{\varepsilon^{\prime}}:=\left\{\omega \in \Omega_{N}^{\text {per }}:\left|x_{u}-u\right|<\varepsilon^{\prime} \text { and }\left|\alpha_{u}\right|<\varepsilon^{\prime} \forall u \in V\right\} .
$$

We claim that

$$
U_{\mathcal{\varepsilon}^{\prime}} \subseteq\left\{H_{N} \in\left[0, c_{3} \delta\left|\Lambda_{N}\right| / 8\right]\right\} .
$$

To prove this, let $\omega=\left(x_{u}, e^{i \alpha_{u}}\right)_{u \in V} \in U_{\varepsilon^{\prime}}$. Then, for any $(u, v) \in E$,

$$
\left|x_{u}-x_{v}-e^{i \alpha_{u}}(u-v)\right| \leq\left|x_{u}-u\right|+\left|v-x_{v}\right|+\left|1-e^{i \alpha_{u}}\right| \cdot|u-v|<3 \varepsilon^{\prime} ;
$$

here we used that $\left|1-e^{i \alpha_{u}}\right| \leq\left|\alpha_{u}\right|$ and $|u-v|=1$. Consequently, using the abbreviation (2.6), the last estimate implies

$$
H_{N}(\omega)=\sum_{u \in \Lambda_{N}} \sum_{\substack{v \in V: \\(u, v) \in E}} h\left(x_{u}-x_{v}-e^{i \alpha_{u}}(u-v)\right) \leq 6\left|\Lambda_{N}\right| c_{1}\left(3 \varepsilon^{\prime}\right)<\left|\Lambda_{N}\right| c_{3} \delta / 8
$$

the last inequality follows by our choice of $\varepsilon^{\prime}$. Hence, (3.28) holds. Consequently,

$$
\begin{aligned}
\rho_{N}\left[H_{N} \in\left[0, c_{3} \delta\left|\Lambda_{N}\right| / 8\right]\right] & \geq \rho_{N}\left[U_{\varepsilon^{\prime}}\right] \\
& \geq\left(\pi\left(\varepsilon^{\prime}\right)^{2}\right)^{\left|\Lambda_{N}\right|-1}\left(2 \varepsilon^{\prime}\right)^{\left|\Lambda_{N}\right|} \geq e^{c_{4}\left|\Lambda_{N}\right|}
\end{aligned}
$$

with $c_{4}=c_{4}\left(\varepsilon^{\prime}\right)=\log \left(2 \pi\left(\varepsilon^{\prime}\right)^{3}\right)$. Inserting this bound into (3.26) yields the claim of the lemma.

Lemma 3.4. Let $c_{3}$ be as in Lemma 3.2 and $c_{1}$ as in (2.6). For all $\delta>0, \beta>0$, and $N \in \mathbb{N}$, one has

$$
\int_{c_{3} \delta\left|\Lambda_{N}\right| / 2<H_{N}<\infty} H_{N} e^{-\beta H_{N}} d \rho_{N} \leq\left(\pi \varepsilon^{2}\right)^{-1} \exp \left(\left|\Lambda_{N}\right|\left\{6 c_{1}(\varepsilon)-\frac{\beta c_{3} \delta}{4}+\frac{1}{2} \log \frac{2 \pi^{3} \varepsilon^{4}}{c_{3} \beta}\right\}\right) .
$$

Note that for large $\beta$, the leading term in the exponent in the claimed upper bound is $-\beta c_{3} \delta\left|\Lambda_{N}\right| / 4$. This is twice the leading part of the exponent in the lower bound (3.25) of the partition sum. The comparison between these two leading terms plays an important role below in the estimate (3.37).

Proof of Lemma 3.4. Here is the rough idea. Using the key lower bound from Lemma 3.2, the integration over the $\alpha$-variables is bounded by a gaussian integral. The remaining integration over the $x$-variables is bounded by a power of the area of the disks associated to the constraints.

Recall that $c_{1}(\varepsilon)<\infty$ by (2.7). It follows from the definition of the Hamiltonian $H_{N}$ that $H_{N} \leq 6\left|\Lambda_{N}\right| c_{1}(\varepsilon) \leq \exp \left(6\left|\Lambda_{N}\right| c_{1}(\varepsilon)\right)$ holds on the set $\left\{H_{N}<\infty\right\}$. Consequently, using exponential Chebyshev in the first step and applying Lemma 3.2 in the last step, we obtain

$$
\begin{aligned}
& \int_{c_{3} \delta\left|\Lambda_{N}\right| / 2<H_{N}<\infty} H_{N} e^{-\beta H_{N}} d \rho_{N} \\
\leq & \int_{H_{N}<\infty} H_{N} e^{\beta\left(H_{N}-c_{3} \delta\left|\Lambda_{N}\right| / 2\right) / 2} \cdot e^{-\beta H_{N}} d \rho_{N} \\
\leq & \exp \left(\left\{6 c_{1}(\varepsilon)-\beta c_{3} \delta / 4\right\}\left|\Lambda_{N}\right|\right) \int_{H_{N}<\infty} e^{-\beta H_{N} / 2} d \rho_{N} \\
\leq & \exp \left(\left\{6 c_{1}(\varepsilon)-\beta c_{3} \delta / 4\right\}\left|\Lambda_{N}\right|\right) \int_{H_{N}<\infty} \exp \left(-\frac{c_{3} \beta}{2} \sum_{u \in \Lambda_{N}} \alpha_{u}^{2}\right) d \rho_{N} .
\end{aligned}
$$


Next, we evaluate the last integral: Denote by $B(x, r)$ the ball centered at $x$ with radius $r$. Enumerate the vertices in $\Lambda_{N}$ as $u_{1}, u_{2}, \ldots, u_{\left|\Lambda_{N}\right|}$ in such a way that $\left(u_{i}, u_{i+1}\right) \in E$ for all $i$ and $u_{\left|\Lambda_{N}\right|}=0$. Consider $\omega \in \Omega_{N}^{\text {per }}$ with $H_{N}(\omega)<\infty$. By the description (3.3) of the set $\left\{H_{N}<\infty\right\}$, for every $u \in \Lambda_{N}$, the component $x_{u}$ is contained in the intersection of the six balls $B\left(x_{v}+e^{i \alpha_{u}}(u-v), \varepsilon\right)$, where $v$ runs through the neighbors of $u$. Consequently, to obtain an upper bound for the contribution to the integral coming from the integration over the $x$-variables, we can proceed as follows: we integrate successively $x_{u_{i}}, i=1,2, \ldots,\left|\Lambda_{N}\right|-1$ over $B\left(x_{u_{i+1}}+e^{i \alpha_{u_{i+1}}}\left(u_{i+1}-u_{i}\right), \varepsilon\right)$. Since $x_{0}=0$, the integration over $x_{u_{\left|\Lambda_{N}\right|}}$ yields no contribution. All other $x_{u}$-integrations give as a factor the Lebesgue measure of a ball of radius $\varepsilon$, namely $\pi \varepsilon^{2}$. Hence,

$$
\begin{aligned}
\int_{H_{N}<\infty} \exp \left(-\frac{c_{3} \beta}{2} \sum_{u \in \Lambda_{N}} \alpha_{u}^{2}\right) d \rho_{N} & \leq\left(\pi \varepsilon^{2}\right)^{\left|\Lambda_{N}\right|-1} \prod_{u \in \Lambda_{N}} \int_{\mathbb{R}} \exp \left(-\frac{c_{3} \beta}{2} \alpha_{u}^{2}\right) d \alpha_{u} \\
& \leq\left(\pi \varepsilon^{2}\right)^{\left|\Lambda_{N}\right|-1}\left(\frac{2 \pi}{c_{3} \beta}\right)^{\left|\Lambda_{N}\right| / 2}
\end{aligned}
$$

Inserting this bound in (3.33) yields the claim of the lemma.

Proof of Theorem 2.3. Let $\delta>0$. First, we rewrite the variance of $\alpha_{0}$ using the properties of the law of the $\alpha_{u}$ 's from Lemma 3.1, then we insert the bound from Lemma 3.2 for the Hamiltonian:

$$
\begin{aligned}
\operatorname{Var}_{\mu_{\beta, N}}\left(\alpha_{0}\right) & =\frac{1}{\left|\Lambda_{N}\right|} \sum_{u \in \Lambda_{N}} \operatorname{Var}_{\mu_{\beta, N}}\left(\alpha_{u}\right) \\
& =\frac{1}{\left|\Lambda_{N}\right|} E_{\mu_{\beta, N}}\left[\sum_{u \in \Lambda_{N}} \alpha_{u}^{2}\right] \leq \frac{1}{c_{3}\left|\Lambda_{N}\right|} E_{\mu_{\beta, N}}\left[H_{N}\right] .
\end{aligned}
$$

To bound the last expectation, we split the domain of integration into two parts, using that $H_{N}<\infty$ holds $\mu_{\beta, N}$-almost surely:

$$
E_{\mu_{\beta, N}}\left[H_{N}\right]=E_{\mu_{\beta, N}}\left[H_{N} 1_{\left\{H_{N} \leq c_{3} \delta\left|\Lambda_{N}\right| / 2\right\}}\right]+\frac{1}{Z_{\beta, N}} \int_{c_{3} \delta\left|\Lambda_{N}\right| / 2<H_{N}<\infty} H_{N} e^{-\beta H_{N}} d \rho_{N} .
$$

The first summand is bounded by $c_{3} \delta\left|\Lambda_{N}\right| / 2$. Denote the second summand in (3.36) by $I$. Inserting the bound from Lemma 3.3 for some $\varepsilon^{\prime} \in\left(0, \pi^{-1 / 2}\right)$ with $c_{1}\left(3 \varepsilon^{\prime}\right)<c_{3} \delta / 48$, and the bound from Lemma 3.4, we obtain:

$$
\begin{aligned}
I & \leq\left(\pi \varepsilon^{2}\right)^{-1} \exp \left(\left|\Lambda_{N}\right|\left\{-c_{4}\left(\varepsilon^{\prime}\right)+\frac{\beta c_{3} \delta}{8}+6 c_{1}(\varepsilon)-\frac{\beta c_{3} \delta}{4}+\frac{1}{2} \log \frac{2 \pi^{3} \varepsilon^{4}}{c_{3} \beta}\right\}\right) \\
& =\left(\pi \varepsilon^{2}\right)^{-1} \exp \left(\left|\Lambda_{N}\right|\left\{c_{5}\left(\varepsilon^{\prime}, \varepsilon\right)-\frac{\beta c_{3} \delta}{8}-\frac{1}{2} \log \left(c_{3} \beta\right)\right\}\right)
\end{aligned}
$$

with $c_{5}\left(\varepsilon^{\prime}, \varepsilon\right)=-c_{4}\left(\varepsilon^{\prime}\right)+6 c_{1}(\varepsilon)+\log \sqrt{2 \pi^{3} \varepsilon^{4}}$.

Note that the leading contribution $-\left|\Lambda_{N}\right| \beta c_{3} \delta / 4$ coming from Lemma 3.4 is only partially 
cancelled by the leading contribution $\left|\Lambda_{N}\right| \beta c_{3} \delta / 8$ from Lemma 3.3. For $\beta>\beta_{0}:=$ $\max \left\{1 / c_{3}, 8 c_{5}\left(\varepsilon^{\prime}, \varepsilon\right) /\left(c_{3} \delta\right)\right\}$, one obtains

$$
I \leq\left(\pi \varepsilon^{2}\right)^{-1} \leq \frac{c_{3}}{2} \delta\left|\Lambda_{N}\right|
$$

for all sufficiently large $N$, namely for $N \geq N_{0}:=\left(c_{3} \delta \pi \varepsilon^{2} / 2\right)^{-1 / 2}$. Inserting this bound into (3.36) yields

$$
E_{\mu_{\beta, N}}\left[H_{N}\right] \leq c_{3} \delta\left|\Lambda_{N}\right|
$$

Hence, (3.35) implies the claim $\operatorname{Var}_{\mu_{\beta, N}}\left(\alpha_{0}\right) \leq \delta$.

\section{Infinite-volume arguments}

In this section, we use tightness arguments to pass to the infinite-volume limit.

Lemma 4.1. The finite-dimensional marginals of $\mu_{\beta, N}, N \in \mathbb{N}$, are tight. As a consequence, there exists a strictly increasing sequence $\left(N_{k}\right)_{k \in \mathbb{N}}$ of natural numbers such that the finite-dimensional marginals of $\left(\mu_{\beta, N_{k}}\right)_{k \in \mathbb{N}}$ converge weakly to the marginals of a limiting distribution $\mu_{\beta}$ on $\Omega_{V}$.

Proof. By the definition of $\mu_{\beta, N}$, we have for any finite edge set $F \subset E$ :

$$
\mu_{\beta, N}\left[\forall e=(u, v) \in F:\left|x_{u}-x_{v}-\gamma_{u}(u-v)\right| \leq \varepsilon\right]=1 .
$$

Consequently, $\left|x_{u}-x_{v}\right| \leq 1+\varepsilon$ holds $\mu_{\beta, N}$-almost surely for all $(u, v) \in E$. Since $x_{0}=0$, this implies $\left|x_{u}\right| \leq \operatorname{dist}(u, 0)(1+\varepsilon)$ for all $u \in V \mu_{\beta, N}$-almost surely, where dist $(u, 0)$ denotes the graph distance between $u$ and 0 in the triangular lattice $\mathscr{T}$. In particular, the finite-dimensional marginals of $\mu_{\beta, N}$ are tight. Thus, using a diagonal sequence argument, there exists a strictly increasing sequence $\left(N_{k}\right)_{k \in \mathbb{N}}$ of natural numbers such that the finite-dimensional marginals of $\mu_{\beta, N_{k}}$ converge weakly to the corresponding marginals of a probability measure $\mu_{\beta}$ on $\Omega_{V}$.

In order to see that any infinite-volume limit $\mu_{\beta}$ as in Lemma 4.1 is non-degenerate, we need to show that no mass of the measures $\mu_{\beta, N}$ can accumulate in the boundaries $\left|x_{u}-x_{v}-\gamma_{u}(u-v)\right|=\varepsilon$ of the admissible domain. More generally, the following lemma shows that no mass of the measures $\mu_{\beta, N}$ can accumulate on null sets with respect to the reference measure. The proof is based on an entropy argument.

Lemma 4.2. For all finite connected $\Lambda \subset V$ with $0 \in \Lambda$ and all $\beta>0$, there exist constants $c_{6}(\beta, \varepsilon)>0$ and $c_{7}(\Lambda, \varepsilon)>0$ such that for all $N$ large enough and all measurable $A \subseteq \Omega_{\Lambda}$ with $0<\rho_{\Lambda}[A]<c_{7}$, one has

$$
\mu_{\beta, N}\left[\omega_{\Lambda} \in A\right] \leq \frac{c_{6}}{-\log \frac{\rho_{\Lambda}[A]}{c_{7}}} .
$$


Proof. Let us first explain the rough idea. The lower bound for the partition sum from Lemma 3.3 gives us an upper bound for the free energy per unit volume, and in turn an upper bound for the entropy (with the mathematics sign convention) per unit volume. This is used to derive an upper bound for the entropy of the system restricted to a fixed volume. Given this entropy bound, the thermal measure cannot give too much mass to sets of small reference measure.

Fix a finite connected set $\Lambda \subset V$ with $0 \in \Lambda$, and let $T$ be an (undirected) spanning tree of $\Lambda$. Let $N$ be so large that $\Lambda$ is contained in the box $\Lambda_{N}$. Take $B_{N, \Lambda}$ to be a maximal subset of $\Lambda_{N}$ with $0 \in B_{N, \Lambda}$ such that the sets $b+\Lambda, b \in B_{N, \Lambda}$, are pairwise disjoint subsets of $\Lambda_{N}$. Note that there is a constant $c_{8}(\Lambda)>0$ such that for all large enough $N$ one has

$$
\left|B_{N, \Lambda}\right| \geq c_{8}\left|\Lambda_{N}\right|
$$

Let $b+T$ denote the translation of the tree $T$ by $b$. Furthermore, let $T_{N}$ be a spanning tree of $\Lambda_{N}$ that contains all trees $b+T, b \in B_{N, \Lambda}$. Define

$$
\begin{aligned}
& \tilde{\Omega}_{N}:=\left\{\left(x_{u}, \gamma_{u}\right)_{u \in V} \in \Omega_{N}^{\mathrm{per}}:\left|x_{v}-x_{u}\right| \leq 1+\varepsilon \text { for all }(u, v) \in T_{N}\right\}, \\
& \tilde{\Omega}_{\Lambda}:=\left\{\left(x_{u}, \gamma_{u}\right)_{u \in \Lambda} \in \Omega_{\Lambda}:\left|x_{v}-x_{u}\right| \leq 1+\varepsilon \text { for all }(u, v) \in T\right\}
\end{aligned}
$$

here we have identified the trees $T_{N}$ and $T$ with their edge sets. By (3.3), all configurations $\omega \in \Omega_{N}^{\text {per }}$ with $H_{N}(\omega)<\infty$ are contained in the set $\tilde{\Omega}_{N}$. In particular, the thermal measure $\mu_{\beta, N}$ is supported on $\tilde{\Omega}_{N}$.

Recall the definition (2.4) of the reference measure $\rho_{N}=\rho_{\Lambda_{N}}$. In the remainder of this proof, we work with the reference measure

$$
\tilde{\rho}_{N}(d \omega):=\frac{1_{\tilde{\Omega}_{N}}(\omega)}{\rho_{N}\left(\tilde{\Omega}_{N}\right)} \rho_{N}(d \omega)=\frac{1_{\tilde{\Omega}_{N}}(\omega)}{\left(\pi(1+\varepsilon)^{2}\right)^{\left|\Lambda_{N}\right|-1}(2 \pi)^{\left|\Lambda_{N}\right|}} \rho_{N}(d \omega) .
$$

The normalization is chosen in such a way that $\tilde{\rho}_{N}$ is a probability measure. Changing the normalization of the reference measure does not change the finite-volume Gibbs measure $\mu_{\beta, N}$, it changes only the normalizing constant $Z_{\beta, N}$ defined in (2.18). The new normalizing constant

$$
\tilde{Z}_{\beta, N}:=\int_{\Omega_{N}^{\text {per }}} e^{-\beta H_{N}} d \tilde{\rho}_{N}
$$

satisfies

$$
\tilde{Z}_{\beta, N}=\frac{Z_{\beta, N}}{\left(\pi(1+\varepsilon)^{2}\right)^{\left|\Lambda_{N}\right|-1}(2 \pi)^{\left|\Lambda_{N}\right|}} \geq \frac{Z_{\beta, N}}{\left(2 \pi^{2}(1+\varepsilon)^{2}\right)^{\left|\Lambda_{N}\right|}} .
$$

By Lemma 3.3, there exists a constant $c_{9} \in \mathbb{R}$ such that $Z_{\beta, N} \geq \exp \left(c_{9}\left|\Lambda_{N}\right|\right)$ holds. (In the terminology of Lemma 3.3, we can use $c_{9}=c_{4}-\beta c_{3} \delta / 8$ for any admissible choice of $\delta$ and $\varepsilon^{\prime}$.) Hence, it follows that

$$
\tilde{Z}_{\beta, N} \geq \exp \left(-c_{10}\left|\Lambda_{N}\right|\right)
$$

with $-c_{10}:=c_{9}-\log \left(2 \pi^{2}(1+\varepsilon)^{2}\right)$.

By the definition (2.17) of $\mu_{\beta, N}$ and the positivity of the Hamiltonian $H_{N}$, we have

$$
\log \frac{d \mu_{\beta, N}}{d \tilde{\rho}_{N}}=-\beta H_{N}-\log \tilde{Z}_{\beta, N} \leq-\log \tilde{Z}_{\beta, N} .
$$


We take the expectation in the last inequality and insert the bound (4.9) for the partition sum to obtain the following estimate for the relative entropy:

$$
E_{\mu_{\beta, N}}\left[\log \frac{d \mu_{\beta, N}}{d \tilde{\rho}_{N}}\right] \leq-\log \tilde{Z}_{\beta, N} \leq c_{10}\left|\Lambda_{N}\right| .
$$

Since relative entropies between probability measures are non-negative, we know $c_{10} \geq 0$.

Let $\lambda$ denote the distribution of $\omega_{\Lambda}$ with respect to $\tilde{\rho}_{N}$, i.e.

$$
\lambda\left(d \omega_{\Lambda}\right)=\frac{1_{\tilde{\Omega}_{\Lambda}}}{c_{7}} \rho_{\Lambda}\left(d \omega_{\Lambda}\right)
$$

with $c_{7}=\rho_{\Lambda}\left[\tilde{\Omega}_{\Lambda}\right]=\left(\pi(1+\varepsilon)^{2}\right)^{|\Lambda|-1}(2 \pi)^{|\Lambda|}$. For this proof, $\lambda$ serves as a reference probability measure on $\Omega_{\Lambda}$. We define

$$
\begin{aligned}
\psi_{N}: \Omega_{N}^{\text {per }} & \rightarrow \Omega_{\Lambda}^{B_{N, \Lambda}}, \\
\left(x_{u}, \gamma_{u}\right)_{u \in V} & \mapsto\left(\left(x_{b+u}-x_{b}, \gamma_{b+u}\right)_{u \in \Lambda}\right)_{b \in B_{N, \Lambda}}
\end{aligned}
$$

Let

$$
\Pi_{\beta, N}:=\psi_{N}\left[\mu_{\beta, N}\right]
$$

denote the image of $\mu_{\beta, N}$ under $\psi_{N}$, and let $\Pi_{\beta, N}^{b}, b \in B_{N, \Lambda}$, denote its marginals on $\Omega_{\Lambda}$. Furthermore, let

$$
\zeta_{N}:=\psi_{N}\left[\tilde{\rho}_{N}\right]
$$

be the image of the reference measure $\tilde{\rho}_{N}$ under $\psi_{N}$. Note that $\zeta_{N}=\lambda^{B_{N, \Lambda}}$ is a product measure. The mathematical relative entropy can only decrease if we replace the measures by their image measure with respect to $\psi_{N}$. Hence, we get

$$
E_{\mu_{\beta, N}}\left[\log \frac{d \mu_{\beta, N}}{d \tilde{\rho}_{N}}\right] \geq E_{\psi_{N}\left[\mu_{\beta, N}\right]}\left[\log \frac{d \psi_{N}\left[\mu_{\beta, N}\right]}{d \psi_{N}\left[\tilde{\rho}_{N}\right]}\right]=E_{\Pi_{\beta, N}}\left[\log \frac{d \Pi_{\beta, N}}{d \zeta_{N}}\right] .
$$

The last relative entropy can only decrease if we replace $\Pi_{\beta, N}$ by the product measure $\tilde{\Pi}_{\beta, N}:=$ $\prod_{b \in B_{N, \Lambda}} \Pi_{\beta, N}^{b}$ with the same marginals as $\Pi_{\beta, N}$. Since the distribution $\mu_{\beta, N}$ is invariant under the translation $\left(x_{u}, \gamma_{u}\right)_{u \in V} \mapsto\left(x_{u+b}-x_{b}, \gamma_{u+b}\right)_{u \in V}$ for any $b \in V$, all marginals $\Pi_{\beta, N}^{b}$ of $\Pi_{\beta, N}$ are equal. Consequently,

$$
\begin{aligned}
E_{\Pi_{\beta, N}}\left[\log \frac{d \Pi_{\beta, N}}{d \zeta_{N}}\right] & \geq E_{\tilde{\Pi}_{\beta, N}}\left[\log \frac{d \tilde{\Pi}_{\beta, N}}{d \zeta_{N}}\right] \\
& =\sum_{b \in B_{N, \Lambda}} E_{\Pi_{\beta, N}^{b}}\left[\log \frac{d \Pi_{\beta, N}^{b}}{d \lambda}\right]=\left|B_{N, \Lambda}\right| E_{\Pi_{\beta, N}^{b}}\left[\log \frac{d \Pi_{\beta, N}^{b}}{d \lambda}\right]
\end{aligned}
$$

for any $b \in B_{N, \Lambda}$. Combining the last inequality with (4.11) and (4.16), we obtain

$$
c_{10}\left|\Lambda_{N}\right| \geq\left|B_{N, \Lambda}\right| E_{\Pi_{\beta, N}^{b}}\left[\log \frac{d \Pi_{\beta, N}^{b}}{d \lambda}\right]
$$


for any $b \in B_{N, \Lambda}$. Hence, by (4.3), we get

$$
\frac{c_{10}}{c_{8}} \geq E_{\Pi_{\beta, N}^{b}}\left[\log \frac{d \Pi_{\beta, N}^{b}}{d \lambda}\right]
$$

for all sufficiently large $N$.

Fix a measurable set $A \in \Omega_{\Lambda}$ with $0<\lambda(A)<1$. Since the relative entropy can only decrease if we take the image measure with respect to the indicator function $1_{A}$ of the set $A$, it follows that

$$
\begin{aligned}
& E_{\Pi_{\beta, N}^{b}}\left[\log \frac{d \Pi_{\beta, N}^{b}}{d \lambda}\right] \geq E_{1_{A}\left[\Pi_{\beta, N}^{b}\right]}\left[\log \frac{d 1_{A}\left[\Pi_{\beta, N}^{b}\right]}{d 1_{A}[\lambda]}\right] \\
& =\Pi_{\beta, N}^{b}[A] \log \frac{\Pi_{\beta, N}^{b}[A]}{\lambda[A]}+\Pi_{\beta, N}^{b}\left[A^{c}\right] \log \frac{\Pi_{\beta, N}^{b}\left[A^{c}\right]}{\lambda\left[A^{c}\right]} \\
& \geq-\frac{2}{e}-\Pi_{\beta, N}^{b}[A] \log \lambda[A]-\Pi_{\beta, N}^{b}\left[A^{c}\right] \log \lambda\left[A^{c}\right] \geq-\frac{2}{e}-\Pi_{\beta, N}^{b}[A] \log \lambda[A] ;
\end{aligned}
$$

here we used that $x \log x \geq-1 / e$ for all $x>0$, and $\lambda\left[A^{c}\right] \leq 1$, since $\lambda$ is a probability measure. Combining (4.19) and (4.20), we obtain the following inequality:

$$
\mu_{\beta, N}\left[\omega_{\Lambda} \in A\right]=\Pi_{\beta, N}^{b}[A] \leq \frac{c_{6}}{-\log \lambda[A]}=\frac{c_{6}}{-\log \frac{\rho_{\Lambda}[A]}{c_{7}}}
$$

with $c_{6}=c_{10} / c_{8}+2 / e$; note that $-\log \lambda[A]>0$.

Corollary 4.3. Let $\mu_{\beta}$ be an infinite-volume limiting distribution as in Lemma 4.1.

(a) The finite-dimensional marginals $\mu_{\beta}\left[\omega_{\Lambda} \in \cdot\right]$ are absolutely continuous with respect to the reference measures $\rho_{\Lambda}$.

(b) For all $(u, v) \in E$, the bound $\left|x_{u}-x_{v}-\gamma_{u}(u-v)\right|<\varepsilon$ holds $\mu_{\beta}$-almost surely.

(c) The local partition sums $Z_{\beta, \Lambda}\left(\omega_{\partial \Lambda}\right)$ arising in (2.11) are strictly positive for $\mu_{\beta}$-almost all $\omega$.

Proof. (a) Let $N \subset \Omega_{\Lambda}$ be a null set with respect to $\rho_{\Lambda}$. Then for every $\delta>0$, there is an open set $A_{\delta} \subseteq \Omega_{\Lambda}$ with respect to the natural topology on $\Omega_{\Lambda}$ such that $N \subseteq A_{\delta}$ and $0<\rho_{\Lambda}\left[A_{\delta}\right] \leq \delta$. Since the marginals of a subsequence $\left(\mu_{\beta, N_{k}}\right)_{k \in \mathbb{N}}$ converge weakly to the corresponding marginals of $\mu_{\beta}$ by Lemma 4.1, we conclude for all small $\delta$, using the bound (4.2):

$$
\begin{aligned}
\mu_{\beta}\left[\omega_{\Lambda} \in N\right] \leq \mu_{\beta}\left[\omega_{\Lambda} \in A_{\delta}\right] & \leq \liminf _{k \rightarrow \infty} \mu_{\beta, N_{k}}\left[\omega_{\Lambda} \in A_{\delta}\right] \\
& \leq \frac{c_{6}}{-\log \frac{\rho_{\Lambda}\left[A_{\delta}\right]}{c_{7}}} \leq \frac{c_{6}}{-\log \frac{\delta}{c_{7}}} \stackrel{\delta \downarrow 0}{\longrightarrow} 0 .
\end{aligned}
$$

Thus, $\mu_{\beta}\left[\omega_{\Lambda} \in N\right]=0$.

(b) We note that for all $(u, v) \in E$, the weaker bound $\left|x_{u}-x_{v}-\gamma_{u}(u-v)\right| \leq \varepsilon$ holds $\mu_{\beta, N}$-almost surely for all $N$. Since the set of all $\left(x_{u}, \gamma_{u}, x_{v}, \gamma_{v}\right)$ fulfilling this bound is closed, the same bound holds also $\mu_{\beta}$-almost surely. Furthermore, the set of all $\left(x_{u}, \gamma_{u}, x_{v}, \gamma_{v}\right)$ fulfilling $\left|x_{u}-x_{v}-\gamma_{u}(u-v)\right|=\varepsilon$ is a 
null set with respect to $\rho_{\{u, v\}}$. Using part (a), this set is also a null set with respect to the marginal of the measure $\mu_{\beta}$ on $\Omega_{\{u, v\}}$. Together, this implies part (b).

Part (c) is an immediate consequence of part (b), since $Z_{\beta, \Lambda}\left(\omega_{\partial \Lambda}\right)$ is strictly positive for all $\omega \in \Omega_{V}$ with $\left|x_{u}-x_{v}-\gamma_{u}(u-v)\right|<\varepsilon$ for all $(u, v) \in E$.

Proof of Theorem 2.4. Fix $\beta>0$, a finite set $\Lambda \subset V$, and take $N \in \mathbb{N}$ large enough. We know the DLR conditions (2.11) for the finite-volume Gibbs measures $\mu_{\beta, N}$ instead of $\mu_{\beta}$. We rewrite these conditions in integral form as follows: For every finite $\Sigma \subset V$ with $\Lambda \cup \partial \Lambda \subseteq \Sigma$, every bounded and continuous function $f: \Omega_{\Sigma} \rightarrow \mathbb{R}$, and every sufficiently large $N$, writing $\mu_{\beta, N, \Sigma}$ for the marginal of $\mu_{\beta, N}$ on $\Omega_{\Sigma}$, one has

$$
\begin{aligned}
& \int_{\Omega_{\Sigma}} Z_{\beta, \Lambda}\left(\chi_{\partial \Lambda}\right) f\left(\chi_{\Sigma}\right) \mu_{\beta, N, \Sigma}\left(d \chi_{\Sigma}\right) \\
& =\int_{\Omega_{\Sigma}} \int_{\Omega_{\Lambda}} f\left(\omega_{\Lambda} \chi_{\Sigma \backslash \Lambda}\right) e^{-\beta H_{\Lambda}\left(\omega_{\Lambda} \mid \chi_{\partial \Lambda}\right)} \rho_{\Lambda}\left(d \omega_{\Lambda}\right) \mu_{\beta, N, \Sigma}\left(d \chi_{\Sigma}\right) .
\end{aligned}
$$

Substituting the definition (2.9) of $Z_{\beta, \Lambda}\left(\chi_{\partial \Lambda}\right)$ and taking the difference of both sides, this is equivalent to

$$
\int_{\Omega_{\Sigma}} \int_{\Omega_{\Lambda}}\left[f\left(\chi_{\Sigma}\right)-f\left(\omega_{\Lambda} \chi_{\Sigma \backslash \Lambda}\right)\right] e^{-\beta H_{\Lambda}\left(\omega_{\Lambda} \mid \chi_{\partial \Lambda}\right)} \rho_{\Lambda}\left(d \omega_{\Lambda}\right) \mu_{\beta, N, \Sigma}\left(d \chi_{\Sigma}\right)=0 .
$$

Now, since the potential $h$ is continuous almost everywhere on the set $\{a \in \mathbb{C}:|a|<\varepsilon\}$, the integrand in (4.24)

$$
\Omega_{\Lambda} \times \Omega_{\Sigma} \ni\left(\omega_{\Lambda}, \chi_{\Sigma}\right) \mapsto\left[f\left(\chi_{\Sigma}\right)-f\left(\omega_{\Lambda} \chi_{\Sigma \backslash \Lambda}\right)\right] e^{-\beta H_{\Lambda}\left(\omega_{\Lambda} \mid \chi_{\partial \Lambda}\right)}
$$

is almost everywhere continuous with respect to $\rho_{\Lambda} \times \rho_{\Sigma}$. Writing $\mu_{\beta, \Sigma}$ for the marginal of $\mu_{\beta}$ on $\Omega_{\Sigma}$, part (a) of Corollary 4.3 implies that $\rho_{\Lambda} \times \mu_{\beta, \Sigma}$ is absolutely continuous with respect to $\rho_{\Lambda} \times \rho_{\Sigma}$. Hence, the function in (4.25) is also almost everywhere continuous with respect to $\rho_{\Lambda} \times \mu_{\beta, \Sigma}$. As this function is bounded, we can pass to the infinite-volume limit in (4.24):

$$
\int_{\Omega_{\Sigma}} \int_{\Omega_{\Lambda}}\left[f\left(\chi_{\Sigma}\right)-f\left(\omega_{\Lambda} \chi_{\Sigma \backslash \Lambda}\right)\right] e^{-\beta H_{\Lambda}\left(\omega_{\Lambda} \mid \chi_{\partial \Lambda}\right)} \rho_{\Lambda}\left(d \omega_{\Lambda}\right) \mu_{\beta, \Sigma}\left(d \chi_{\Sigma}\right)=0 .
$$

This identity together with the positivity of the local partition sums (part (c) of Corollary 4.3) is equivalent to the DLR condition (2.11).

Proof of Theorem 2.1. By Theorem 2.4, the $\mu_{\beta, N_{k}}$-distribution of $\gamma_{0}=e^{i \alpha_{0}}$ converges weakly as $k \rightarrow$ $\infty$ to the $\mu_{\beta}$-distribution of $\gamma_{0}$. Since $e^{i \alpha} \mapsto \alpha \in(-\pi, \pi]$ is discontinuous only at the point $e^{i \alpha}=-1$ and $\mu_{\beta}\left[\gamma_{0}=-1\right]=0$, Lemma 3.1 implies

$$
E_{\mu_{\beta}}\left[\alpha_{0}\right]=\lim _{k \rightarrow \infty} E_{\mu_{\beta, N_{k}}}\left[\alpha_{0}\right]=0 .
$$

Using Theorem 2.3 , we conclude that for $\delta>0$, we have

$$
\lim _{\beta \rightarrow \infty} \operatorname{Var}_{\mu_{\beta}}\left(\alpha_{0}\right)=\lim _{\beta \rightarrow \infty} \lim _{k \rightarrow \infty} \operatorname{Var}_{\mu_{\beta}, N_{k}}\left(\alpha_{0}\right) \leq \delta .
$$

Hence, it follows that $\lim _{\beta \rightarrow \infty} \operatorname{Var}_{\mu_{\beta}}\left(\alpha_{0}\right)=0$. 
Sketch of proof of Remark 2.2. Given $\varepsilon, \Lambda$, and $\varphi$ as in Remark 2.2, we fix $R>\max _{u \in \Lambda}|u|$, and take a smooth function $f:[0, \infty) \rightarrow[0,1]$ with $f(r)=1$ for $0 \leq r \leq R, f(r)=0$ for all large $r$, and $\sup _{r>0}(r+1)\left|f^{\prime}(r)\right|<\varepsilon / \varphi$. Taking $\log r$ times a negative constant close to 0 , and translating and truncating this function appropriately, one can see that such a function $f$ exists. We set for $u \in V$ and $-\varphi \leq t \leq \varphi$

$$
x_{u}(t)=e^{i t f(|u|)} u, \quad \gamma_{u}(t)=e^{i t f(|u|)}
$$

For this choice, the properties (a), (c), (d), and (e) are obviously true. To see the bound in (b), we estimate for $t \in[-\varphi, \varphi]$ and $(u, v) \in E$, for some value $r$ between $|u|$ and $|v|$, using ||$u|-| v|| \leq 1$ :

$$
\begin{aligned}
& \left|x_{u}(t)-x_{v}(t)-\gamma_{u}(t)(u-v)\right|=|v| \cdot\left|e^{i t f(|u|)}-e^{i t f(|v|)}\right| \leq|v| \cdot|t| \cdot|f(|u|)-f(|v|)| \\
& \leq \varphi \cdot|v| \cdot\left|f^{\prime}(r)\right| \leq \varphi \cdot(r+1)\left|f^{\prime}(r)\right|<\varepsilon .
\end{aligned}
$$

Acknowledgement We would like to thank two anonymous referees for making useful suggestions.

\section{References}

[Aiz94] M. Aizenman. On the slow decay of $\mathrm{O}(2)$ correlations in the absence of topological excitations: remark on the Patrascioiu-Seiler model. J. Statist. Phys., 77(1-2):351-359, 1994. MR1300539

[BLRW06] L. Bowen, R. Lyons, C. Radin, and P. Winkler. A solidification phenomenon in random packings. SIAM J. Math. Anal., 38(4):1075-1089 (electronic), 2006. MR2274475

[DS75] R. L. Dobrushin and S. B. Shlosman. Absence of breakdown of continuous symmetry in two-dimensional models of statistical physics. Comm. Math. Phys., 42:31-40, 1975. MR0424106

[FP81] J. Fröhlich and C. Pfister. On the absence of spontaneous symmetry breaking and of crystalline ordering in two-dimensional systems. Comm. Math. Phys., 81(2):277-298, 1981. MR0632763

[FP86] J. Fröhlich and C.-E. Pfister. Absence of crystalline ordering in two dimensions. Comm. Math. Phys., 104(4):697-700, 1986. MR0841677

[FSS76] J. Fröhlich, B. Simon, and T. Spencer. Infrared bounds, phase transitions and continuous symmetry breaking. Comm. Math. Phys., 50(1):79-95, 1976. MR0421531

[Geo99] H.-O. Georgii. Translation invariance and continuous symmetries in two-dimensional continuum systems. In Mathematical results in statistical mechanics (Marseilles, 1998), pages 53-69. World Sci. Publ., River Edge, NJ, 1999. MR1886241

[ISV02] D. Ioffe, S. Shlosman, and Y. Velenik. 2D models of statistical physics with continuous symmetry: the case of singular interactions. Comm. Math. Phys., 226(2):433-454, 2002. MR1892461 
[Mer68] N.D. Mermin. Crystalline order in two dimensions. Phys. Rev., 176(1):250-254, 1968.

[MW66] N.D. Mermin and H. Wagner. Absence of ferromagnetism or antiferromagnetism in oneor two-dimensional isotropic Heisenberg models. Phys. Rev. Lett., 17(22):1133-1136, 1966.

[NH79] D. R. Nelson and B. I. Halperin. Dislocation-mediated melting in two dimensions. Phys. Rev. B, 19(5):2457-2484, 1979.

[Pfi81] C. E. Pfister. On the symmetry of the Gibbs states in two-dimensional lattice systems. Comm. Math. Phys., 79(2):181-188, 1981. MR0612247

[Ric05] T. Richthammer. Two-dimensional Gibbsian point processes with continuous spin symmetries. Stochastic Process. Appl., 115(5):827-848, 2005. MR2132600

[Ric09] T. Richthammer. Translation invariance of two-dimensional Gibbsian systems of particles with internal degrees of freedom. Stochastic Process. Appl., 119(3):700-736, 2009. MR2500256 